\title{
Topological distribution of reversible and non-reversible degradation in perovskite solar cells
}

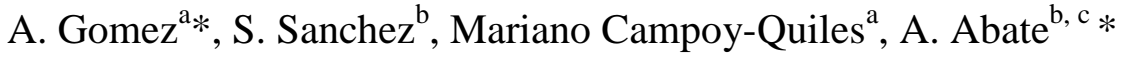 \\ a Institut de Ciència de Materials de Barcelona (ICMAB-CSIC), Campus UAB, 08193 \\ Bellaterra, Spain. \\ ${ }^{\mathrm{b}}$ Adolphe Merkle Institute, University of Fribourg, Chemin des Verdiers 4, CH-1700 \\ Fribourg, Switzerland \\ c Helmholtz-Zentrum Berlin für Materialien und Energie, Kekuléstrasse 5, 12489 \\ Berlin, Germany
}

*Corresponding author: A G agomez@icmab.es ; AA antonio.abate@helmholtz$\underline{\text { berlin.de }}$

Keywords: Perovskite solar cell; photovoltaics; degradation; solar energy; AFM 


\section{$\underline{\text { ABSTRACT }}$}

Lead halide perovskites have recently raised as an easy to process and cost-effective photovoltaic material. However, stability issues have to be addressed to meet the market need for 25 years durable technology. The stability of the perovskite itself, as well as the stability of the perovskite embedded in a complete device under real working conditions, are a key challenge for perovskite solar cells.

Within this study, we used Photoconductive Atomic Force Microscopy (pcAFM) and Photoluminescence imaging (PL) to investigate at the nanoscale level the degradation of the perovskite film under light and voltage stress. Then, we correlate the nanoscale pcAFM and PL analysis to the macroscopic device behaviour in similar ageing condition. We found that non-reversible performance losses in a complete device originate from degradation localised at the grain boundaries of the perovskite film. Interesting, within the bulk of the perovskite grains we observed fully reversible behaviours. We conclude that the grain boundaries are detrimental to the device stability and they need to be minimised or passivated to achieve fully stable perovskite solar cells even under anhydrous conditions. 


\section{$\underline{\text { INTRODUCTION }}$}

Halide perovskites are currently attracting large attention as novel photovoltaic material to design the next generation of solar cells. ${ }^{1}$ Despite their relatively recent discovery, lab-scale perovskite solar cells (PSCs) with power conversion efficiencies (PCEs) above $22 \%$ proved the potential of perovskites as photovoltaic materials. ${ }^{2}$ PSCs are a serious candidate to compete or to work in tandem with established technologies such as silicon and cadmium telluride solar cells. ${ }^{3}$

The next and probably more difficult challenge for PSCs is to stabilise their PCE for 25 years equivalent standard working conditions. Accelerated 1 year equivalent ageing test, such as 1000 hours under maximum power point tracking at 1 sun simulated illumination, showed that the most stable lab-scale PSCs so far reported still lose about $10 \%$ of their initial efficiency compared to less than $0.5 \%$ of the market availed silicon modules. More stable PSCs were reported, but their initial PCE was far below the stateof-the-art. ${ }^{4}$ While the stability gap with the silicon seems huge, recent works demonstrated that PSCs recover part of their losses after a rest in dark. ${ }^{5,6}$ This has been associated with the migration of ionic defects within the perovskite film and their accumulation at the interface with the selective charge contacts in the device. ${ }^{7,8}$ In addition to reversible losses, non-reversible ones may originate from degradation of the perovskite film. Water and oxygen are well-known sources of perovskite film degradation. Several studies indicated that PSCs exposed to water and oxygen under illumination lose their initial PCE in relatively short time. ${ }^{9-15}$ More recently, Wang et al. demonstrated that the perovskite grain boundaries are the access point for water entering and diffusing within the perovskite film. ${ }^{16}$ They showed that the grain boundaries within the perovskite film initiate the degradation, which successively propagates towards the grain bulk. The work of Wang et al. highlighted the importance of reducing or passivating the grain boundaries to improve the stability in the humid environment during the preparation of the device. Nevertheless, the impact of the grain boundaries on the stability in a condition resembling a solar cell operational, i.e. under illumination and load, required still further investigation.

In this work, we show that under light and voltage stress the grain boundaries initiate the degradation of the perovskite film. We make use of Photoconductive Atomic Force Microscopy (pcAFM) and photoluminescence (PL) mapping to 
investigate the degradation at the nanoscale. Correlating the pcAFM and PL nanoscale analysis to macroscopic devices, we found that non-reversible losses in power conversion efficiency originate from perovskite degradation localised at the grain boundaries. Notably, the bulk of the perovskite grains exhibits fully reversible behaviours. We conclude that the grain boundaries are a source of instability and thus they need to be minimised or passivated to achieve fully stable perovskite solar cells. 


\section{$\underline{\text { RESULTS AND DISCUSSION }}$}

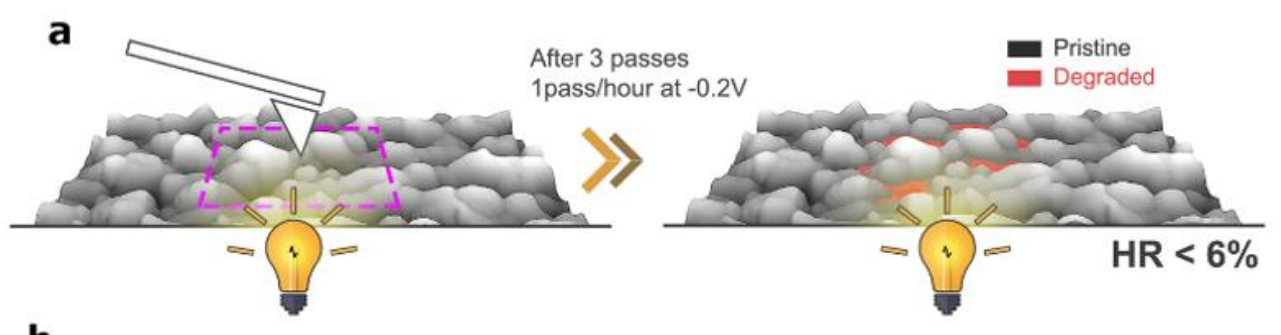

b
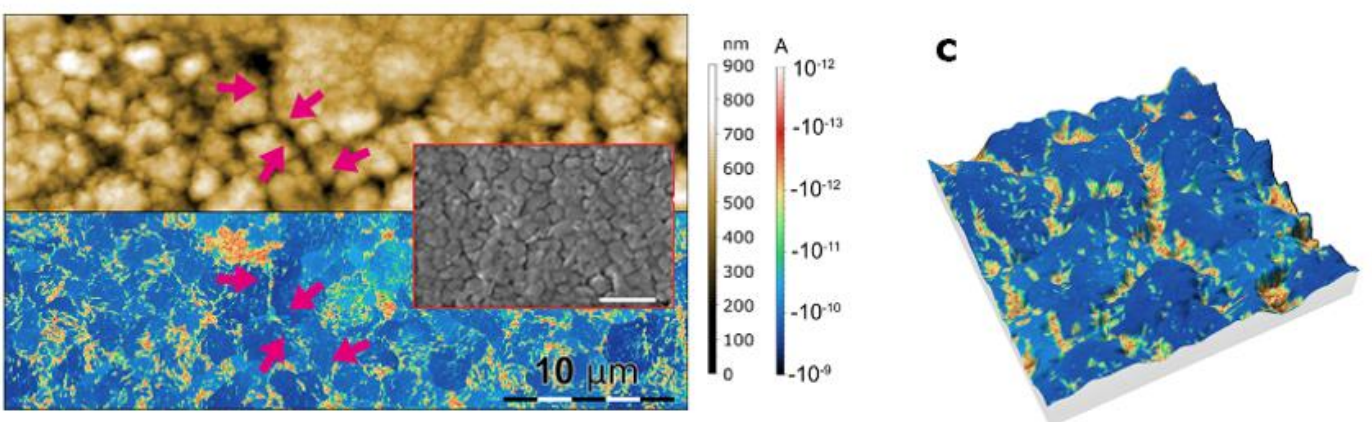

d
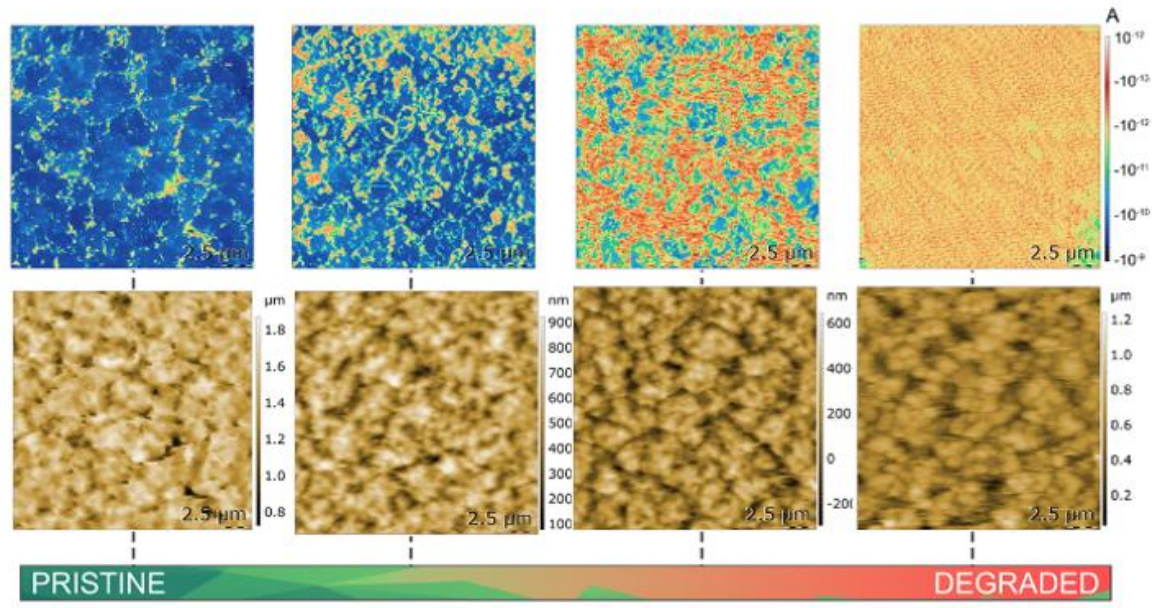

$\mathrm{OH}$

$24 \mathrm{H}$

$28 \mathrm{H}$

$96 \mathrm{H}$

e

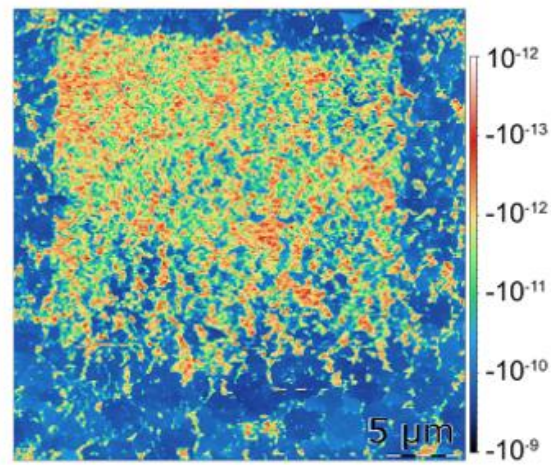

Figure 1. a. Schematic of the measurement protocol: the perovskite film surface was scanned using pcAFM while the sample was illuminated from the FTO substrate with Red-Green-Blue LEDs, 625-630, 517-520, 465-470 nm, each of them with $1.2 \mathrm{~mW}$ of power. $-0.2 \mathrm{~V}$ load was applied between the FTO and the tip. During the whole 
experiment, the samples were kept under dry air with a relative humidity below $6 \%$. b. Topography (top), corresponding pcAFM current (bottom) and SEM image inset, the arrows highlight the grain boundaries. c. 3D view superimposing current colour image and topography. d. pcAFM maps (top) and their corresponding topography images (bottom) collected from different virgin areas of the perovskite film, along different times during the light exposure. e. pcAFM map of the same sample where a square of $20 \mu \mathrm{m} \times 20 \mu \mathrm{m}$ was recorded by performing three passes with a $-0.2 \mathrm{~V}$ biased tip and waiting one hour between each of the scans.

To investigate the perovskite film degradation at nanometer level we performed Photoconductive Atomic Force Microscopy (pcAFM). ${ }^{17}$ Figure 1a depicts a schematic of the measurement protocol. The samples were kept continuously under illumination in dry air with a relative humidity lower than $6 \%$ to avoid a fast degradation mechanism induced by high humidity. ${ }^{18}$ More details of the measurement setup are reported in S1.

Before discussing the effect of the applied voltage on the degradation, we show the high-resolution pcAFM image to correlate the AFM topography of the perovskite film and the current distribution (see Figure 1b). A SEM image is also included to show that the same topography is observed as extracted by AFM. The arrows in Figure $1 \mathrm{~b}$ highlight the perovskite grain boundaries, which can be identified in the both AFM and the pcAFM images. The correlation between grain boundaries on the current distribution is possibly clearer in the $3 \mathrm{D}$ plot in Figure 1c, which overlaps film topography and current mapping. We collected both the trace and retraced images to rule out artefacts that may arise from bad mechanical contact (see S2) and we repeated the same experiment with smaller grain perovskite films (see S3).

In the first set of measurements (Figure 1d), we mapped different virgin areas of the same perovskite film at different times during the light exposure. The specific scan was $512 \times 512$ pixels, while the tip speed was maintained constant at a pace of $20 \mu \mathrm{m} \mathrm{s}^{-1}$, leading to a frame image time of $29 \mathrm{~min}$. $-0.2 \mathrm{~V}$ bias was applied between the FTO substrate and the tip during the scan. Comparing the photocurrent maps (top) with the topographic images (bottom), we can observe that the photocurrent is distributed uniformly inside the perovskite grains with discontinuities matching the grain boundaries. Notably, the effect of the boundaries propagates from the edges towards the bulk of the grains, incorporating the entire surface in few days under illumination. Control experiments, carried out turning on and off the light during the same scan, 
demonstrated that the recorded current comes from photogenerated charge carriers (see S4).

In the second set of measurements (Figure 1e), we scanned multiple times a smaller portion of the same area with the aim to investigate the impact of the applied voltage in addition to the light exposure. These testing conditions are closer the complete device operation, where the perovskite film experiences an externally applied and built-in voltage under illumination. The experiment was completed in about two hours, thus in a significantly shorter time than the previous experiment in Figure 1d, which occurs on a timescale of days. It can be observed (Figure 1e) that, under the combination of the voltage and light, the effect of the grain boundaries in 2 hours resembles that observed after 24 hours under illumination but without bias. In both experiments, the average current recorded in the pristine samples was about $810^{-11} \mathrm{~A}$, and it dropped below $410^{-}$ ${ }^{12}$ A after few days under solo illumination or after few hours under illumination and applied voltage. The current noise calculated from Figure $1 \mathrm{c}$ is $810^{-13} \mathrm{~A}$, which is two orders of magnitude lower than the value recorded. The impact of the voltage is even clearer if we compare in Figure $2 \mathrm{f}$ the internal area, which was scanned multiple times, with the external area scanned only once. The external area resembles a freshly made sample, while the internal one, which was longer exposed to the applied voltage, shows the grain boundary effect. Thus, the voltage accelerates the propagation of the grain boundaries effect.

While Wang et al. reported a similar study in humid air, ${ }^{16}$ we can here exclude that the propagation of the grain boundary effect is due to water diffusing from the boundaries into the bulk of the perovskite grains. First, because the humidity was taken constantly lower than $6 \%$, while perovskite film hydration required over $30 \%$ relative humidity, ${ }^{11}$, 16 and second because the measured RMS roughness has no significant changes during the experiment, which suggests no significant hydration. ${ }^{11}$ We measured 130 and 142 nm RMS before and after the experiment, while the hydration is reported to double up the RMS roughness of perovskite films. ${ }^{11}$ 

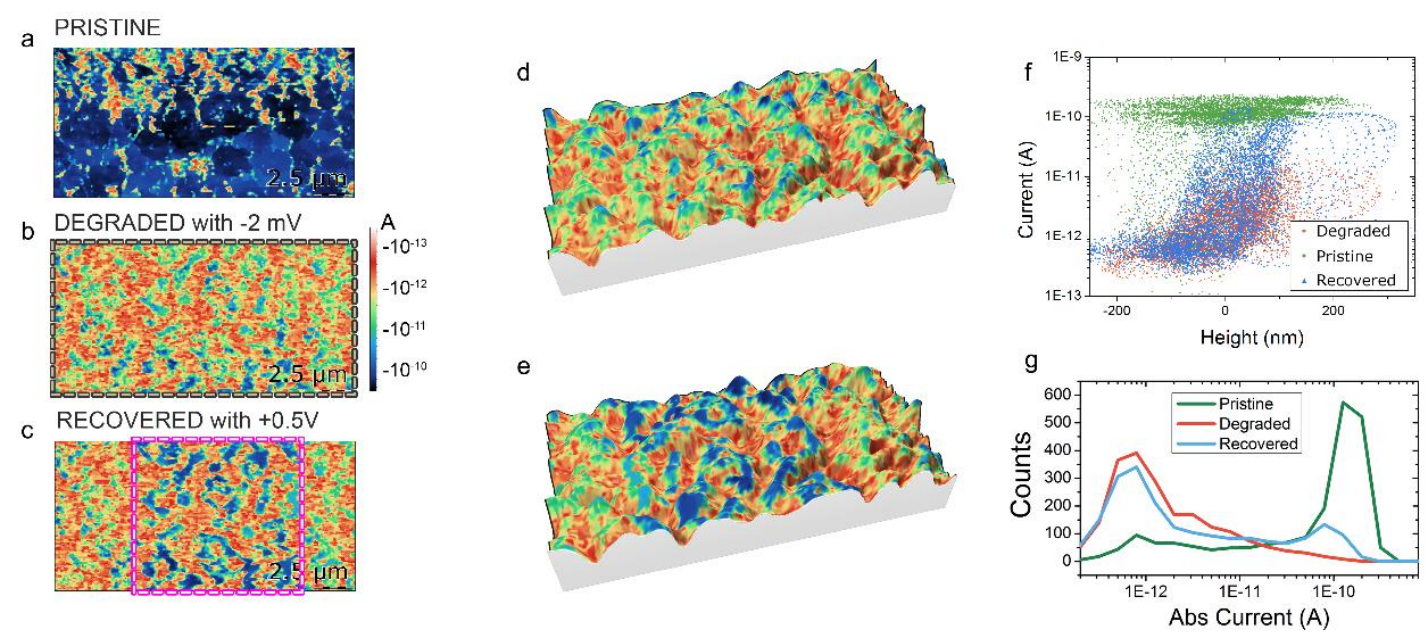

Figure 2. 2D Photoconductive Atomic Force Microscopy (pcAFM) for the same areas scanned multiple times as described in Figure 1: a pcAFM map of the pristine sample, $\mathbf{b}$ the same area after stress under negative applied bias and $\mathbf{c}$ after stress under a positive applied bias (scale unit in Ampere). Figure $\mathbf{d}$ and e corresponding 3D analysis, where the photocurrent overlap with the topography, $\mathbf{f}$ Graph reporting the photocurrent as a function of the surface height for the measurements in a, b, and c. g Histogram is reporting the counts as a function of the photocurrent values.

Several recent works have shown that PSCs degraded in real working condition, i.e. applied load under illumination, can in part recover their initial power conversion efficiency. ${ }^{5-7}$ To investigate if the voltage mediated photocurrent degradation was to some extent reversible, we tried to regenerate the sample applying a reverse voltage.

First, we mapped the photocurrent of a random area of the perovskite film, and then we accelerated the degradation of the sample under light and voltage $(-2 \mathrm{mV})$, similar to what we described for the experiment in Figure 1. Thus, we applied a reverse voltage $(0.5 \mathrm{~V})$ on a smaller area within the one previously scanned (degraded). The photocurrent maps of the pristine, degraded and recovered samples are reported in Figure $2 \mathrm{a}, \mathrm{b}$ and $\mathrm{c}$. In addition to the $2 \mathrm{D}$ distribution of the photocurrent, we reported a $3 \mathrm{D}$ analysis overlapping the photocurrent to the AFM topography, see Figure $2 \mathrm{~d}$ and $2 \mathrm{e}$.

From the photocurrent maps, it looks clear that there is a partial recovery of the photocurrent within the area where the reverse voltage was applied and almost no difference for the control area (on the sides), where no regenerating voltage was applied. Furthermore, the 3D analysis shows that the inner part of the perovskite grains, 
corresponding to the top of the hills in the topographic images, fully recovers the initial photocurrent while the grain boundaries, corresponding to the valleys in the topographic images, remain entirely degraded(additional data is provided in SI, see S5). This is clearer from the graph in Figure 2f, where we report the photocurrent as a function of the surface height. We can observe that the initial photocurrent is rather uniformly distributed; conversely, the recovered photocurrent is clearly higher on the top of the hills. Similar spatial inhomogeneity was reported by Garrett et al. for the open circuit voltage distribution within the perovskite grains. ${ }^{19}$

To quantify the regeneration, histograms of the photocurrent maps were reported in Figure $2 \mathrm{~g}$. Each count corresponds to a pixel of the photocurrent image. We calculated that the sample recovered $35 \%$ of the initial photocurrent. Interestingly, we can see a sharp separation between the degraded and non-degraded current peaks, which indicates the presence of a net-front of degradation moving from the boundaries to the bulk of the grain. This is in line with what recently reported by Sun et al., ${ }^{15}$ who proposed that degradation initiating at the boundaries and slowly diffusing towards the bulk of the perovskite grains is due to the presence of trace amount of oxygen. They argued that, under illumination, the perovskite injects electrons within molecular oxygen generating highly reactive superoxide species, which decompose the perovskite. We want to highlight that this process is evidently non-reversible and thus unrelated to the reversible behaviours we observed.

To clarify if the reversible behaviour is a volume or a surface effect, we performed AFM scratching experiments, increasing the force on the tip to penetrate the surface of the perovskite film. Scanning a degraded area with the scratching experiment (see S7 of SI), we found that the initial photocurrent is fully recovered if the force on the tip is strong enough to penetrate few nanometers from the surface. This is compatible with the model proposed by Richardson et al., ${ }^{20}$ where ions composing the perovskite can migrate and accumulate within a few nanometers at the interface with the selective contacts. Here, we infer that ions accumulating at the interface with the tip screens the applied voltage, which results in a lower photocurrent. Therefore, when the tip penetrates the charged layer, the original photocurrent is recovered. Piezoelectric effect is not compatible with a constant current all over the mapped image, as we are employing a constant force to perform the scans (Nature Communications 8, Article number: 1113 (2017) doi:10.1038/s41467-017-01361-2). 
a
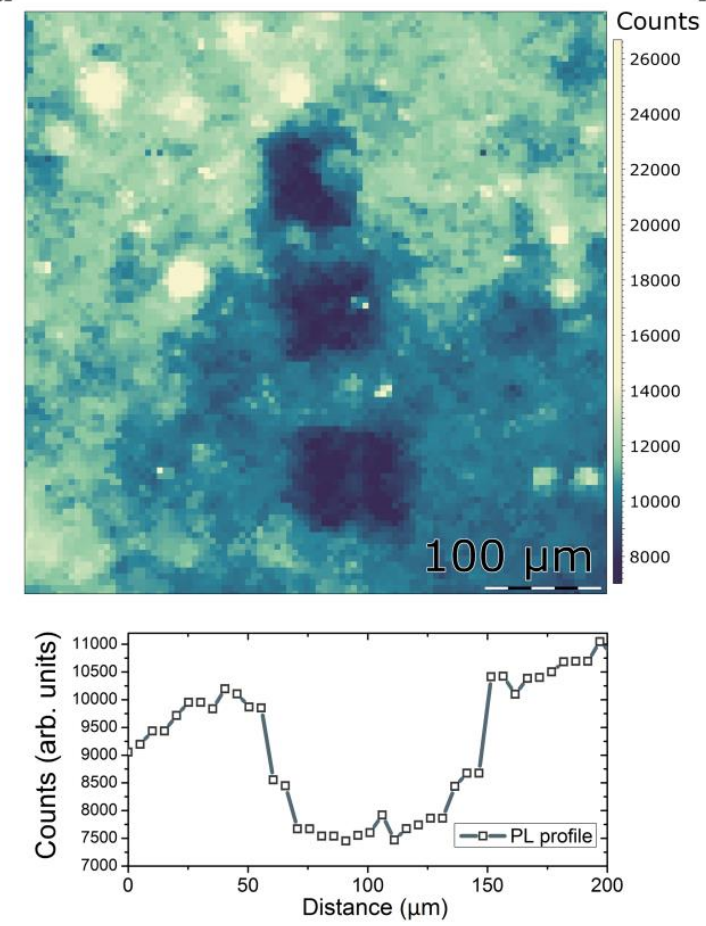

b
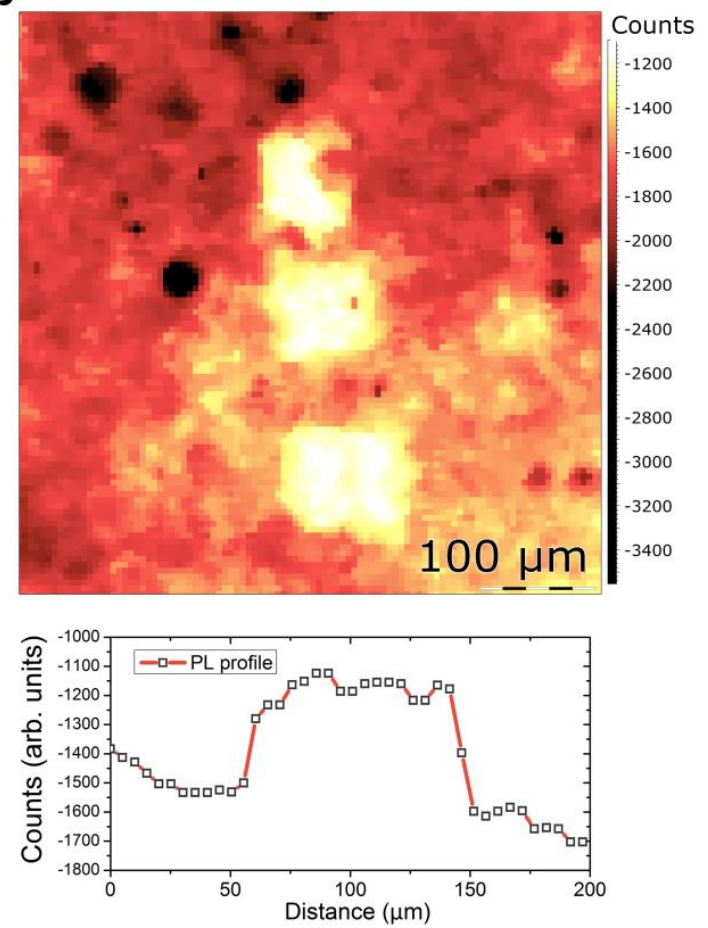

Figure 3. Photoluminescence (a) and reflectance (b) images of a sample that was previously degraded using the AFM under bias, describing three degraded squares within an otherwise not degraded film.

To provide further insights into the degradation mechanisms upon illumination and biasing of the perovskite, we made use of Raman/photoluminescence (PL) microscopy. We measured a sample that had been previously degraded in three $100 \mu \mathrm{m}$ x $100 \mu \mathrm{m}$ squared areas using an AFM under light and applied voltage. We recorded the Raman/PL signal upon laser excitation at $785 \mathrm{~nm}$ as well as the reflectivity signal (the reflection of the laser itself at zero Raman shift). Outside the degraded area, the signal is dominated by the large PL from the perovskite with no clear Raman features, as reported elsewhere. ${ }^{21}$ Within the degraded area, the PL slightly decreases. Importantly, no signature of strong chemical degradation is observed. For instance, no Raman peaks of lead iodide or other species, which typically have very clear and strong vibrational fingerprint, are detected in those areas. Raman can typically detect several chemicals if the relative volume fractions are greater than a few $\%$. Degradation occurring mainly at surfaces and grain boundaries may then not be accessible using this technique. On the other hand, the $785 \mathrm{~nm}$ laser is reflected more strongly in the degraded areas (Figure 3b). A possible explanation could be that the surface is becoming more reflective due to a decrease in surface roughness; however, the AFM data in Figures 1 and 2 do not 
support this hypothesis. An alternative explanation is that the material itself becomes more reflective. Bulk degradation would typically result in the lower refractive index, ${ }^{22}$ which would reduce the normal incidence reflectivity. Instead, an increase in surface conductivity would have the opposite effect through an enhanced Drude tail of absorption. This is well in agreement with the aforementioned scratching experiments, which suggest that the degradation of photoconductivity is associated with the voltage screening resulting from the accumulation of charges (ions) at the interface. Incidentally, the increase in reflectance means that less light is available for absorption at the perovskite, which helps to explain the reduction in PL. Free charges could also act as PL quenching centres. In summary, the spectroscopic experiments suggest that the level of chemical degradation is small within these experiments, and it may be localised at grain boundaries and the surface. 


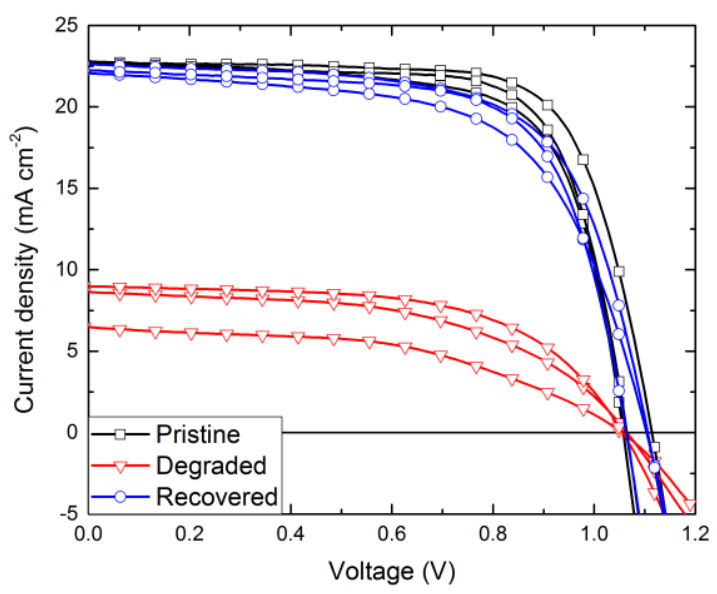

Figure 4. Perovskite solar cells current density-voltage (JV) curves collected before and immediately after 1 -hour forward voltage under 1.5 AM illumination. The sample was then left resting for three days in dark and dry air with a relative humidity below $6 \%$. The $J-V$ curves were measured at a scan rate of $10 \mathrm{mV} \mathrm{s}^{-1}$ from forward bias to short circuit condition under AM1.5 simulated solar light illumination. A shadow mask with an aperture of about $0.1 \mathrm{~cm}^{2}$ defined the active area.

Table 1. Average photovoltaic performance parameters: open-circuit voltage $\left(V_{\mathrm{oc}}\right)$, short circuit current $\left(J_{\mathrm{sc}}\right)$, fill factor $(\mathrm{FF})$ and maximum power conversion efficiency (PCE) extracted from the $J-V$ curves in Figure 4.

\begin{tabular}{ccccc}
$\begin{array}{c}\text { Average } \\
\text { of 3 devices }\end{array}$ & $\begin{array}{c}\boldsymbol{V}_{\text {oc }} \\
(\mathbf{m V})\end{array}$ & $\begin{array}{c}\boldsymbol{J}_{\text {sc }} \\
\left(\mathbf{m A} / \mathbf{c m}^{\mathbf{2}}\right)\end{array}$ & $\begin{array}{c}\mathbf{F F} \\
(\mathbf{\%})\end{array}$ & $\begin{array}{c}\mathbf{P C E} \\
(\mathbf{\%})\end{array}$ \\
\hline Pristine & 1099 & 22.8 & 70 & 17.4 \\
Degraded & 1061 & 8.0 & 53 & 4.6 \\
Recovered & 1055 & 22.3 & 68 & 15.9 \\
\hline
\end{tabular}

In order to corroborate what we observed from the nanoscale analysis of the macroscopic behavior of devices in similar ageing conditions, we prepared complete PSCs following one of the most recent methods, which makes use of mixed halide and mixed cation perovskite films deposited on a thin layer of mesoporous $\mathrm{TiO}_{2}{ }^{23}$ Mimicking the measurement protocol described in Figure 1 for the pcAFM analysis, we measured the current density-voltage curves (JVs) of PSCs before and after 1 hours under forward voltage in light and dry air. Figure 4 displays the JV curves with the corresponding performance parameters listed in Table 1. All the performance parameters decrease after the voltage and light bias test, in particular, the short circuit current $\left(\mathrm{J}_{\mathrm{sc}}\right)$ suffered the most. Then, we left the devices resting in the dark and dry air for about three days before to measure them again. Unexpectedly, all the devices recovered most of their initial performance, with some residual permanent losses in the 
open circuit voltage $\left(\mathrm{V}_{\mathrm{oc}}\right)$. We note that the influence of the hysteresis is rather minimal in all the samples - pristine, degraded and recovered, as reported in S8.

Similar reversible behaviour has been reported for PSCs prepared by other research groups, using different perovskite compositions and device architectures. For example, Tan et al. demonstrated almost fully reversible performance losses in planar PSCs over 500 hours under maximum power point tracking. ${ }^{6}$ Comparable behaviours have been reported by Saliba et al. for PSC employing mesoporous $\mathrm{TiO}_{2}$ with a multiple cation perovskite compositions. ${ }^{5}$ In our previous study, we proposed that such reversible losses are due to the intrinsic perovskite ion migration and their accumulation at the interface with the selective charge contacts. ${ }^{7}$ However, we could not rule out any potential contribution of extrinsic ion migration due to, for example, chemical doping of the contacts, as recently reported by $\mathrm{Li}$ et $a .^{24}$ Here, the photocurrent mapping collected with the pcAFM from perovskites without selective contacts provided a direct evidence that such reversible behaviors are intrinsic to the material and most likely linked to the ion migration.

Comparing the nanoscale and the macroscopic analysis, we can observe that the $\mathrm{J}_{\mathrm{sc}}$ decay in the complete device is almost fully reversible while the initial photocurrent in the pcAFM is only in part recovered (35\%, see Figure 2). Such a difference may originate from having the perovskite film coated with the hole-transporting layer in the complete device and naked in the pcAFM measurement. We believe that the holetransporting material can penetrate in between the small gaps of the perovskite layer, effectively passivating most of the grain boundaries. ${ }^{25}$ This is expected to slow down the degradation in a complete device as compared to a perovskite film directly exposed to air. We would also like to note that when using the AFM tip, the voltage drop is concentrated in a much smaller area (the effective tip area) compared to the full device, which means that the voltage will be much stronger during the AFM experiments, and thus ion migration and photocurrent degradation is likely to be more pronounced. 


\section{CONCLUSIONS}

Perovskite solar cells ageing under real working condition exhibit reversible losses in addition to permanent degradation of the initial power conversion efficiency. We demonstrated that reversible and non-reversible power losses originate from different mechanisms localised within the bulk and at the boundaries of the perovskite grains respectively. Under condition resembling the solar cells operational, i.e. applied load under the light, we demonstrated that non-reversible behaviours correlate with degradation of the perovskite started at the grain boundaries and slowly migrating towards the bulk of the grain. On the contrary, reversible phenomena are associated with the bulk of the perovskite grains, which are more resilient to degradation. Interestingly, we could observe a sharp separation between the inner reversible and outer non-reversible domains, which indicates a net front of degradation slowly moving from the boundaries to the bulk of the grain. Hence, we conclude that the grain boundaries are detrimental for the device stability and thus they need to be minimised, enlarging the grain size, or passivated, coating the perovskite surface, to achieve fully stable perovskite solar cells. 


\section{EXPERIMENTAL PROCEDURE}

Substrate preparation. Photovoltaic devices were fabricated on FTO coated glass (Pilkington). Substrates were cleaned in Hellmanex (Aldrich), then 30 min sonication in Hellmanex, 15 min sonication in IPA, and 5 min of oxygen plasma etching. Then, 30 $\mathrm{nm} \mathrm{TiO}_{2}$ compact layers were deposited on FTO via spray pyrolysis at $450{ }^{\circ} \mathrm{C}$ from a precursor solution of titanium diisopropoxide bis (acetylacetonate) in anhydrous ethanol and acetylacetonate. After the spraying, the FTO substrates were left at $450{ }^{\circ} \mathrm{C}$ for 5 min before cooling down to room temperature. Then, a mesoporous $\mathrm{TiO}_{2}$ layer was deposited by spin coating for $10 \mathrm{~s}$ at $4000 \mathrm{rpm}$ with a ramp of $2000 \mathrm{rpm} \mathrm{s}^{-1}$, using a 30 $\mathrm{nm}$ particle paste (Dyesol $30 \mathrm{NR}-\mathrm{D}$ ) diluted in ethanol to achieve 150-200 nm thick layer. After the spin coating, the FTO substrates were dried at $100{ }^{\circ} \mathrm{C}$ for $10 \mathrm{~min}$, and the films were annealed on a programmable hotplate (2000 W, Harry Gestigkeit GmbH) to crystallise $\mathrm{TiO}_{2}$ at $450{ }^{\circ} \mathrm{C}$ for 30 min under dry airflow. Li-doping of mesoporous $\mathrm{TiO}_{2}$ was accomplished by spin coating a 0.1 M solution of bis(trifluoromethane)sulfonimide lithium salt Li-TFSI in acetonitrile at $3000 \mathrm{rpm}$ for 10 $\mathrm{s}$ followed by another sintering step at $450{ }^{\circ} \mathrm{C}$ for $30 \mathrm{~min}$. After cooling down to 150 ${ }^{\circ} \mathrm{C}$, all the substrates were immediately transferred in a nitrogen atmosphere glove box (with an overpressure varying of 2-3 mbar) for depositing the perovskite films.

Perovskite precursor solutions and film preparation. The mixed cation and mixed halide perovskite films were deposited as previously reported. ${ }^{23}$ The organic salts were purchased from Dyesol; the lead compounds from TCI. The perovskite precursor solutions were deposited from a precursor solution containing FAI (1 M), $\mathrm{PbI}_{2}(1.1 \mathrm{M})$, $\operatorname{MABr}(0.2 \mathrm{M}), \mathrm{PbBr}_{2}(0.22 \mathrm{M})$ and CsI (0.05 M) in anhydrous DMF: DMSO 4:1 (V:V). We note that this composition contains a lead iodide and bromide excess as reported elsewhere. The perovskite solution was spin-coated with a two steps program at 1000 and $6000 \mathrm{rpm}$ for 10 and $20 \mathrm{~s}$ respectively. During the second step, $100 \mu \mathrm{L}$ of chlorobenzene was poured on the spinning substrate $5 \mathrm{~s}$ before the end of the program. The substrates were then annealed at $100{ }^{\circ} \mathrm{C}$ for $1 \mathrm{~h}$ in a nitrogen-filled glove box. For the photocurrent generation studies, perovskite films were deposited directly on FTO substrates using the same perovskite precursor solution. After the perovskite annealing, the substrates were cooled down for few minutes, and a spiro-OMeTAD (Merck) solution (70 mM in chlorobenzene) was spin coated at $4000 \mathrm{rpm}$ for $20 \mathrm{~s}$. SpiroOMeTAD was doped with bis(trifluoromethylsulfonyl)imide lithium salt (Li-TFSI, 
Sigma-Aldrich),

tris(2-(1H-pyrazol-1-yl)-4-tert-butylpyridine)-

cobalt(III)

tris(bis(trifluoromethylsulfonyl)imide) (FK209, Dynamo) and 4-tert-Butylpyridine (TBP, Sigma-Aldrich). The molar ratio of additives for spiro-OMeTAD was $0.5,0.03$ and 3.3 for Li-TFSI, FK209 and TBP respectively. Finally, to complete the devices 80 $\mathrm{nm}$ of the gold top electrode was thermally evaporated under ultrahigh vacuum.

Photovoltaic device testing. For photovoltaic measurements, a solar simulator from ABET Technologies (Model 11016 Sun 2000) with a xenon arc lamp was used, and the solar cell response was recorded using a Metrohm PGSTAT302N Autolab. The intensity of the solar simulator was calibrated to $100 \mathrm{~mW} / \mathrm{cm}^{2}$ using a silicon reference cell from ReRa Solutions (KG5 filtered). $J$ - $V$-curves were measured in reverse and forward bias at a scan rate of $10 \mathrm{mV} / \mathrm{s}$. The external bias at open circuit condition synchronised with the solar simulator (ABET) was performed using a light intensity of $100 \mathrm{~mW} / \mathrm{cm}^{2}$ and a Metrohm PGSTAT302N Autolab.

Photoconductive atomic force microscopy. Photoconductive AFM (pcAFM) was used to explore the degradation mechanisms of the cell. A solid platinum tip, with reference RMN-25PT300, is connected to logarithmic I-to-V converter "Resiscope" while the sample is biased by a user-selectable voltage. The sample is irradiated with a RedGreen-Blue led $(630,520$ and $470 \mathrm{~nm})$ with the same electrical power applied to the three diodes. A constant force of $200 \mathrm{nN}$ was applied to the sample comprising a good electrical contact to collect the generated current guaranteeing no mechanical damage to the sample. To avoid humidity effect, before turning the light on in the box, we lowered the ambient humidity by introducing compressed air into the AFM box.

Photoluminescence mapping. Photoluminescence maps were acquired using a WITec alpha300 confocal Raman imaging system in backscattering geometry exciting with a 785nm solid state laser. Images contained 10.000 spectra, each collected on the flight and with $50 \mathrm{~ms}$ integration time. With the same system, both PL and Raman scattering are collected simultaneously. The chosen excitation wavelength limits the degradation of the sample and enables a relatively small PL background compared to exciting at shorter wavelengths, which facilitate the identification of any emerging species by Raman. This configuration only allows, however, to collect the tail of the PL of the perovskite. The laser reflectance was also collected during the same experiment at a zero Raman shift position. 


\section{ACKNOWLEDGEMENT}

NFFA-Europe has received funding from the EU's H2020 framework programme for research and innovation under grant agreement n. 654360. ICMAB acknowledges financial support from the Spanish Ministry of Economy and Competitiveness, through the "Severo Ochoa" Programme for Centres of Excellence in R\&D (SEV- 2015-0496) and projects ENE2015-68995-REDT and MAT2015-70850-P. 


\section{References}

1. Saliba, M.; Correa-Baena, J.-P.; Graetzel, M.; Hagfeldt, A.; Abate, A. Angewandte Chemie International Edition 2017.

2. Yang, W. S.; Park, B.-W.; Jung, E. H.; Jeon, N. J.; Kim, Y. C.; Lee, D. U.; Shin, S. S.; Seo, J.; Kim, E. K.; Noh, J. H. Science 2017, 356, (6345), 1376-1379.

3. Bush, K. A.; Palmstrom, A. F.; Zhengshan, J. Y.; Boccard, M.; Cheacharoen, R.; Mailoa, J. P.; McMeekin, D. P.; Hoye, R. L. Z.; Bailie, C. D.; Leijtens, T. Nature Energy 2017, 2, 17009.

4. Grancini, G.; Roldán-Carmona, C.; Zimmermann, I.; Mosconi, E.; Lee, X.; Martineau, D.; Narbey, S.; Oswald, F.; De Angelis, F.; Graetzel, M. Nature Communications 2017, 8.

5. Saliba, M.; Matsui, T.; Domanski, K.; Seo, J.-Y.; Ummadisingu, A.; Zakeeruddin, S. M.; Correa-Baena, J.-P.; Tress, W. R.; Abate, A.; Hagfeldt, A. Science 2016, 354, (6309), 206-209.

6. Tan, H.; Jain, A.; Voznyy, O.; Lan, X.; de Arquer, F. P. G.; Fan, J. Z.; Quintero-Bermudez, R.; Yuan, M.; Zhang, B.; Zhao, Y. Science 2017, 355, (6326), 722-726.

7. Domanski, K.; Roose, B.; Matsui, T.; Saliba, M.; Turren-Cruz, S.-H.; Correa-Baena, J.-P.; Carmona, C. R.; Richardson, G.; Foster, J. M.; De Angelis, F. Energy \& Environmental Science 2017, 10, (2), 604-613.

8. Liu, J.; Yin, X.; Liu, X.; Que, M.; Que, W. The Journal of Physical Chemistry C 2017, 1932.

9. Mosconi, E.; Azpiroz, J. M.; De Angelis, F. Chemistry of Materials 2015, 27, (13), 48854892.

10. Aristidou, N.; Sanchez-Molina, I.; Chotchuangchutchaval, T.; Brown, M.; Martinez, L.; Rath, T.; Haque, S. A. Angewandte Chemie International Edition 2015, 54, (28), 8208-8212.

11. Leguy, A. I. M. A.; Hu, Y.; Campoy-Quiles, M.; Alonso, M. I.; Weber, O. J.; Azarhoosh, P.; Van Schilfgaarde, M.; Weller, M. T.; Bein, T.; Nelson, J. Chemistry of Materials 2015, 27, (9), 3397-3407.

12. Song, Z.; Abate, A.; Watthage, S. C.; Liyanage, G. K.; Phillips, A. B.; Steiner, U.; Graetzel, M.; Heben, M. J. Advanced Energy Materials 2016, 6, (19), 1521.

13. Ahn, N.; Kwak, K.; Jang, M. S.; Yoon, H.; Lee, B. Y.; Lee, J.-K.; Pikhitsa, P. V.; Byun, J.; Choi, M. Nature communications 2016, 7, 13422.

14. Aristidou, N.; Eames, C.; Sanchez-Molina, I.; Bu, X.; Kosco, J.; Islam, M. S.; Haque, S. A. Nature Communications 2017, 8.

15. Sun, Q.; Fassl, P.; Becker-Koch, D.; Bausch, A.; Rivkin, B.; Bai, S.; Hopkinson, P. E.; Snaith, H. J.; Vaynzof, Y. Advanced Energy Materials 2017, 1614.

16. Wang, Q.; Chen, B.; Liu, Y.; Deng, Y.; Bai, Y.; Dong, Q.; Huang, J. Energy \& Environmental Science 2017, 10, (2), 516-522.

17. Coffey, D. C.; Reid, O. G.; Rodovsky, D. B.; Bartholomew, G. P.; Ginger, D. S. Nano letters 2007, 7, (3), 738-744.

18. Song, Z.; Abate, A.; Watthage, S. C.; Liyanage, G. K.; Phillips, A. B.; Steiner, U.; Graetzel, M.; Heben, M. J. Advanced Energy Materials 2016, 6, (19\%@ 1614-6840).

19. Garrett, J. L.; Tennyson, E. M.; Hu, M.; Huang, J.; Munday, J. N.; Leite, M. S. Nano Letters 2017, 17, (4), 2554-2560.

20. Richardson, G.; O'Kane, S. E. J.; Niemann, R. G.; Peltola, T. A.; Foster, J. M.; Cameron, P. J.; Walker, A. B. Energy \& Environmental Science 2016, 9, (4), 1476-1485.

21. Leguy, A. M. A.; Goñi, A. R.; Frost, J. M.; Skelton, J.; Brivio, F.; Rodríguez-Martínez, X.; Weber, O. J.; Pallipurath, A.; Alonso, M. I.; Campoy-Quiles, M. Physical Chemistry Chemical Physics 2016, 18, (39), 27051-27066.

22. Leguy, A. M. A.; Frost, J. M.; McMahon, A. P.; Sakai, V. G.; Kochelmann, W.; Law, C.; Li, X.; Foglia, F.; Walsh, A.; O'Regan, B. C. Nature communications 2015, 6.

23. Saliba, M.; Matsui, T.; Seo, J.-Y.; Domanski, K.; Correa-Baena, J.-P.; Nazeeruddin, M. K.; Zakeeruddin, S. M.; Tress, W.; Abate, A.; Hagfeldt, A. Energy \& environmental science 2016, 9, (6), 1989-1997. 
24. Li, Z.; Xiao, C.; Yang, Y.; Harvey, S. P.; Kim, D. H.; Christians, J. A.; Yang, M.; Schulz, P.; Nanayakkara, S. U.; Jiang, C.-S. Energy \& Environmental Science 2017, 10, (5), 1234-1242.

25. Shao, Y.; Xiao, Z.; Bi, C.; Yuan, Y.; Huang, J. Nature communications 2014, 5, 5784 \%@ 2041-1723. 